\title{
La Resurrección del Público en la Autoría de los Procesos Creativos en los Espacios Procomunes
}

\section{The Resurrection of the Public Authorship in the Creative Process of the Common Places}

\author{
Chen YiJu \\ Facultad de Bellas Artes. Universidad Complutense de Madrid. \\ chenaliciayiju@gmail.com
}

Recibido: 22 de noviembre de 2012

Aprobado: 19 de febrero de 2013

\section{Resumen}

"La Resurrección del Público en la Autoría de los Procesos Creativos" se basa en la teoría de la sensibilidad. Trata sobre el auge de la satisfacción como experiencia estética que cada individuo percibe y crea. A diferencia del arte restringido, como la arquitectura, la escultura, la pintura, la música y la poesía, esta experiencia estética que cada uno crea, se acerca más a la vivencia cotidiana.

Actualmente esta forma de entender el arte está presente en muchos espacios procomunes, tales como centros sociales autogestionados y huertos urbanos.

Para construir una epistemología tenemos que recurrir al paradigma "síntoma-paradigmas-estilosejemplos", constituyéndola desde el contexto social y desde el análisis interno sobre el pensamiento y la acción que aporta el poder constituyente de cada individuo. A partir de esta reflexión, podemos observar cómo este modelo social hace que todo el mundo pueda ser artista.

Podemos encontrar en las palabras de Hegel, Schiller, Dewey y en el espíritu del artesano el fundamento para la resurrección del público en la autoría de los procesos creativos.

Finalmente, razono cómo los espacios creados de este modo, tienen un impacto social, ya que últimamente han surgido muchos conceptos como el decrecimiento, el movimiento "grassroot" y el "transicional town".

Palabras Clave: la experiencia estética, espacios procomunes, huertos urbanos, centros sociales autogestionados, espíritu artesano.

Yiju, C. (2013): La Resurrección del Público en la Autoría de los Procesos Creativos en los Espacios Procomunes. Arte, Individuo y Sociedad, 25(3) 508-523

Abstract 


\begin{abstract}
"The resurrection of the public authorship in the creative process " is a concept that I want to propose. It is a concept developed based on the theory of sensitivity. It's about the rise of satisfaction, which we understand as aesthetic experience which every individual perceives and creates. Unlike the arts which tend to be predefined and shaped by the past such as architecture, sculpture, painting, music, poetry, etc., the aesthetic experience is another artistic form closer to our daily experiences.

Currently this way of understanding the art exists in many Common Places, such as self-run autonomous social centers and urban gardens.

To construct an epistemology of this phenomenon in aesthetic study, we must use the paradigm "symptom-paradigms-styles-examples", constituting the knowledge from the social context and from the internal analysis about how the thoughts and the actions bring power to all individuals. From this reflection, this essay shows how this social model makes everyone be artist. This paper reconsiders the internal analysis of aesthetic experience through texts and reflections of Hegel, Schiller and Dewey, in which they had previously debated the spirit of the artisan.

Finally, I reason how spaces created in this way have a social impact, since lately many ideas related to the concept of Degrowth and "transition town" movements have emerged.
\end{abstract}

Key Words: aesthetic experience, Common places, urban farming, self-run autonomous social centers, spirit of artisan.

Yiju, C. (2013): The Resurrection of the Public Authorship in the Creative Process of the Common Places. Arte, Individuo y Sociedad, 25(3) 508-523

Sumario: 1. Introducción: la resurrección del público en la autoría de los procesos creativos en los espacios procomunes, 2. Metodología: Síntoma $\rightarrow$ Paradigma $\rightarrow$ Estilos $\rightarrow$ Ejemplos, 3. Análisis de los síntomas y de las líneas de tendencia sobre los casos investigados, 3.1. La producción y el consumo de arte en el mercado del arte, 3.2. Las artes que se generan en base a la experiencia estética del público, 3.3.El CSA la Tabacalera, 3.4. El Campo de la Cebada, 3.5.Recorrido histórico de cómo es la muerte y la resurrección del autor, 4.Análisis sobre la lógica de ejemplos existentes, 4.1. La relación entre la percepción del sujeto con los casos, 4.2. La relación entre la percepción del sujeto con la teoría, 5. Conclusiones. Referencias.

Este artículo recoge resultados de la investigación de la tesis doctoral "El Valor Estético en el Diseño del Eco-Hábitat" financiada por el Ministerio de Educación de Taiwán.

\title{
1. Introducción: la resurrección del público en la autoría de los procesos creativos en los espacios procomunes
}

Cuando formulé esta hipótesis de cómo es la resurrección del público en la autoría de los procesos creativos a través de la creatividad que cada individuo genera en los espacios procomunes, como por ejemplo, los huertos urbanos y los centros sociales autogestionados, me pregunté, en qué contexto le correspondería y cómo circularía la producción y el consumo del arte en dichos espacios. El desarrollo de esta hipótesis tiene que ver con la estética, es decir, la teoría de lo bello.

Es La analitica kantiana de la experiencia estética desemboca en una inteligente 
concepción de lo bello como «lo que agrada universalmente sin concepto». Al sostener que nuestros juicios de gusto, o juicios estéticos, tienen pretensiones de universalidad que van más allá del capricho individual (Garrido, 2007, p.17).

Cuando la estética se fundó en 1750, se concibió como la teoría de la sensibilidad (por Davide Hume, Edmund Burke, Baugarten, Immanuel Kant). Actualmente, si observamos el mercado del arte, debido a la intervención del mercado, como bien explicó Wendy Griswold (1994, p.15) en el diamante cultural, existen cuatro vértices: el producto artístico, el artista, el consumidor del arte y el contexto social, que se conectan entre sí por seis líneas que marcan el consumo del arte en la sociedad, manteniendo el círculo del consumo y la producción del arte. Así pues, los museos se convierten en cajas blancas que otorgan más valor a las obras artísticas.

La discusión de la experiencia estética se perdió. Los espacios procomunes, son lugares donde aún no se ha introducido el mercado. La experiencia de la construcción en sí, se acerca a la creatividad cotidiana y al trabajo relacionado con los cuidados. El "arte" que se genera es un producto colectivo de una comunidad, fruto de la red de personas cercanas que trabajan en ella, que producen, alimentan y aprecian mutuamente la obra, poseyendo ellos mismos múltiples funciones: autores, críticos, usuarios y espectadores al mismo tiempo.

Esta experiencia generada en espacios procomunes me suscita una inquietud que me hace volver a revisar la historia del arte, entendiendo que la historia no es una acumulación de hechos definitivos, sino múltiples interpretaciones respecto a la actualidad.

En dOCUMENTA (13), una de las reflexiones sobre la "investigación artística" destacada por Carolyn Christov-Bakargiev y Chus Martínez, trata sobre cómo el arte crea perspectivas de "quizás", recuperando el poder escéptico de un sujeto, llevándole a una búsqueda continua de lo real, desafiando lo incuestionable o conocimientos totalitarios.

Dice Chus Martínez:

"Artistic research" is an awful term; it is confusing in its similitude to 'research' as we know it in academic fields. (...). 'Research' here does not name the embodiment of any particular form of academic training, but the gesture of placing the 'maybe' at the core of the real" (Martínez, 2012, p.46).

Los artistas compilan información para formar una hipótesis y con una actitud escéptica en el centro de lo real, haciendo zozobrar el conocimiento establecido. En este artículo, voy a presentar dos espacios procomunes que conozco personalmente y que se encuentran en Madrid, el CSA la Tabacalera y el Campo de Cebada. Considero que el "Quizás" sólo se ha manifestado en estos dos espacios autoconstruidos por los ciudadanos, en los que se muestra que la creatividad la puede tener todo el mundo. En esta discusión sobre la experiencia de la estética, se desvanece el paradigma de diferenciación entre los artistas y los no artistas, teniendo en cuenta que será el público el que constituya sus realidades y sus subjetividades. 


\section{Metodología: Síntoma $\rightarrow$ Paradigma $\rightarrow$ Estilos $\rightarrow$ Ejemplos}

(Villasante, 2002, p. 208).

\begin{tabular}{|c|c|c|c|}
\hline Síntomas & Paradigmas & Estilos & Ejemplos \\
\hline $\begin{array}{l}\text { "Mostrar los } \\
\text { Síntomas de } \\
\text { arranque que uno } \\
\text { toma, concierta } \\
\text { capacidad } \\
\text { auto-crítica, y } \\
\text { reconocer sus } \\
\text { límites desde el } \\
\text { primer momento" } \\
\text { (Villasante, 2002, } \\
\text { p. 208). }\end{array}$ & $\begin{array}{l}\text { “Luego pasa } \\
\text { por razonar las } \\
\text { polémicas lógicas } \\
\text { sobre cada caso, } \\
\text { con referencia } \\
\text { a los distintos } \\
\text { Paradigmas. Y } \\
\text { abrir unas nuevas } \\
\text { posibilidades de } \\
\text { interpretación, } \\
\text { nuevos planos } \\
\text { desde donde mirar } \\
\text { con nuevos ojos” } \\
\text { (Villasante, 2002, p. } \\
\text { 208). }\end{array}$ & $\begin{array}{l}\text { "En tercer lugar } \\
\text { construir Estilos, } \\
\text { posiciones } \\
\text { propias que se } \\
\text { van a adoptar, } \\
\text { justificándolas ante } \\
\text { cada problemática } \\
\text { apuntada, es decir } \\
\text { las estrategias" } \\
\text { (Villasante, 2002, } \\
\text { p. 208). }\end{array}$ & $\begin{array}{l}\text { "Finalmente mostrar } \\
\text { Ejemplos, caminos } \\
\text { que quedan por } \\
\text { recorrer, al menos } \\
\text { justificar los casos } \\
\text { elegidos para } \\
\text { continuar, porque } \\
\text { el final o meta } \\
\text { dificilmente puede } \\
\text { enunciar si somos } \\
\text { un poco prudentes" } \\
\text { (Villasante, 2002, p. } \\
\text { 208). }\end{array}$ \\
\hline $\begin{array}{l}\text { ARTE Y } \\
\text { VIVENCIA } \\
\text { EXPERIENCIA } \\
\text { ESTÉTICA } \\
\text { en } \\
\text { - Huertos } \\
\text { urbanos, } \\
\text {-Centros sociales } \\
\text { autogestionados, } \\
\text { - proyectos } \\
\text { comunitarios } \\
\text { como Transition } \\
\text { Towns. }\end{array}$ & $\begin{array}{l}\text { ANÁLISIS } \\
\text { Hacer reflexiones } \\
\text { generales. } \\
\text { Líneas de } \\
\text { tendencias. }\end{array}$ & $\begin{array}{l}\text { PRAXIS } \\
\text { Coordinación de la } \\
\text { estrategia y de la } \\
\text { táctica. } \\
\text { REDES reflexivas } \\
\text { Aclaración de la } \\
\text { identificación de } \\
\text { casos investigados } \\
\text { y la justificación } \\
\text { respecto a ello con } \\
\text { teorías previas } \\
\text { (reflexiones } \\
\text { existentes) y } \\
\text { experiencias } \\
\text { actuales }\end{array}$ & $\begin{array}{l}\text { FOROS e indicadores } \\
\text { relativos sobre el arte } \\
\text { en este tema } \\
\text { METODOLOGÍAS } \\
\text { INSTITUYENTES Y } \\
\text { CREATIVAS (IAP- } \\
\text { PAI) }\end{array}$ \\
\hline
\end{tabular}

Recurro a esta línea porque el tema que discutimos en este artículo toca la teoría de la sensibilidad, por la cual, es importante tener un método de investigación abierto y participativo. Esta metodología originada de la Investigación-Acción Participativa, no solo tiene el fin de desarrollar modelos simétricos en base a la experiencia vivencial, sino que lleva implícita expresiones del activismo social y un compromiso 
ideológico para contribuir a la praxis (colectiva) del pueblo.

La construcción epistemológica de este esquema es una reflexión y un examen crítico de las opiniones populares. Esta forma de constituir el conocimiento ha sido aplicada ampliamente en estudios antropológicos, por ejemplo, en la "antropología social de apoyo" (Colombres, 1982; Hernández, 1987) y en estudios pedagógicos tales como la educación crítica expandida por Paulo Freire. Esta metodología tiene la potencia de crear una epistemología apropiada para los "pueblos sin historias": "Los etnólogos se están acercando a las culturas nativas y locales con un esquema de referencia participativo, llegando asi más allá de Sol Tax, C. Levi-Strauss y D. Lewis (Stavenhagen, 1986; Bonfil Batalla, 1981)” (Rahman \& Borda, 1992/2009, p.185).

\section{Análisis de los síntomas y de las líneas de tendencia sobre los casos investigados}

1. En los espacios autogestionados o cogestionados, podemos observar otro modo de crear arte, ya que el intercambio no se restringe solo en el circulo de producción y de consumo, sino que también se realiza a través de la oferta de servicios. Si falta la financiación, un artista que trabaja en el proyecto de autoconstrucción tiene que hacerse valer y tiene que compensar su labor con otros medios, por ejemplo, trabajar a cambio de comida y del uso de espacios. En el Campo de Cebada, el arquitecto no sólo diseña los objetos, sino que también ofrece servicios, como el acompañamiento en la gestión, la participación, la vinculación, la intermediación, y los diseños de las asambleas. La experiencia estética que intento esbozar en este artículo se base en la vivencia en la creación de todos los participantes en conjunto. Este modo de hacer, se diferencia de la producción y el consumo de arte en el mercado artístico actual, porque su proceso colectivo cuestiona la autoría de un único autor. La experiencia estética que cada sujeto logra no se puede intercambiar por una firma o un precio. Además, para realizar la virtud de gratitud y de gratuidad que existe en estos procesos, se considera el factor del tiempo y el capital humano. De este modo, lo que se produce tiene un impacto social directo.

2. En este artículo, queremos enfatizar que la estrategia (la manera en que el tema transmitido en el lenguaje artístico porta algo en la autonomía del arte) y la táctica (la absorción del lenguaje social y político en el lenguaje artístico) concuerdan con los ejemplos que citamos: los huertos urbanos y los centros sociales autogestionados. Estas praxis se convierten en un modo de hacer y se integran en la vivencia. Estas micro acciones pueden convertirse en modas y culturas independientemente de la autoría de estas. Esto explica por qué el arte generado es autónomo y contextual, a diferencia de la estética premoderna, cuyo concepto de la belleza universal es diferente. Según los indicadores de la Estética de Diálogo (Kester, 2004), la Investigación- Acción Participativa, los huertos urbanos, los centros sociales autogestionados, las arquitecturas vernáculas, Geniurs Loci, etc., son procesos y productos que estimulan "lo bello" y son artes auténticas. La entrada de una percepción estética no es unidireccional para comunicar lo que ha sido experimentando, sino que es una comunicación intersubjetiva en la percepción y comprensión. 
3. Si lo examinamos a partir de un proceso creativo, la unión de la percepción de lo bello y de la creación, genera una belleza aproximada a lo natural. Richard Sennett (2009, p.278) llamó "linde" a este tipo de espacio, puesto que sus procesos creativos se acercan más a la política del artesano, ya que los patrones de espacio como frutos de intervención, representan la armonía, el caos y la multiculturalidad al mismo tiempo.

\subsection{La producción y el consumo de arte en el mercado del arte}

Según el artista Mel Chin en el seminario Transdisciplinary Lecture: Mel Chin, organizada por The New School en diciembre de 2010, en la actualidad, el arte se segrega de la sociedad en la producción y el consumo de arte.

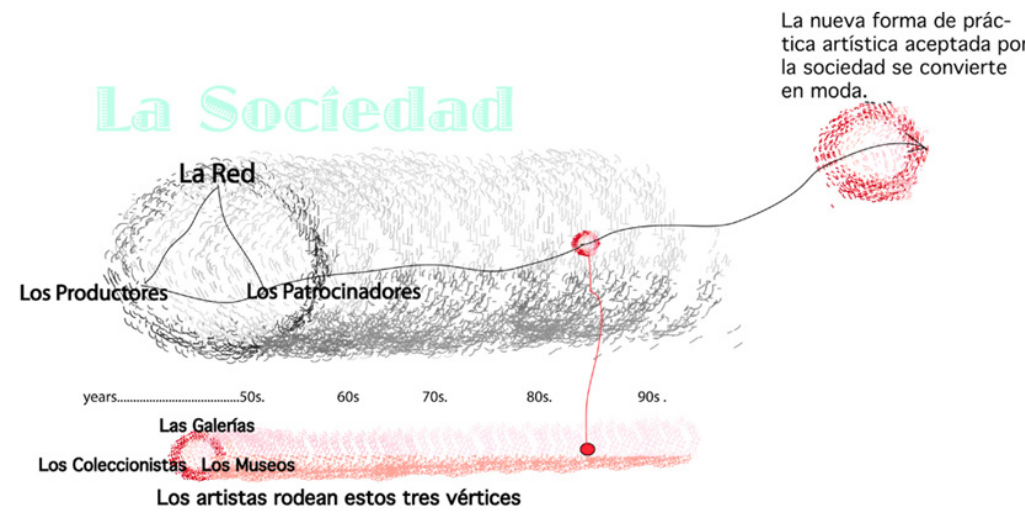

BL CIRCUITO DEL ARTE

Figura 1. La relación entre el arte y la sociedad, Mel Chin (imagen reproducida).

\subsection{La producción y el consumo de arte en el mercado del arte}

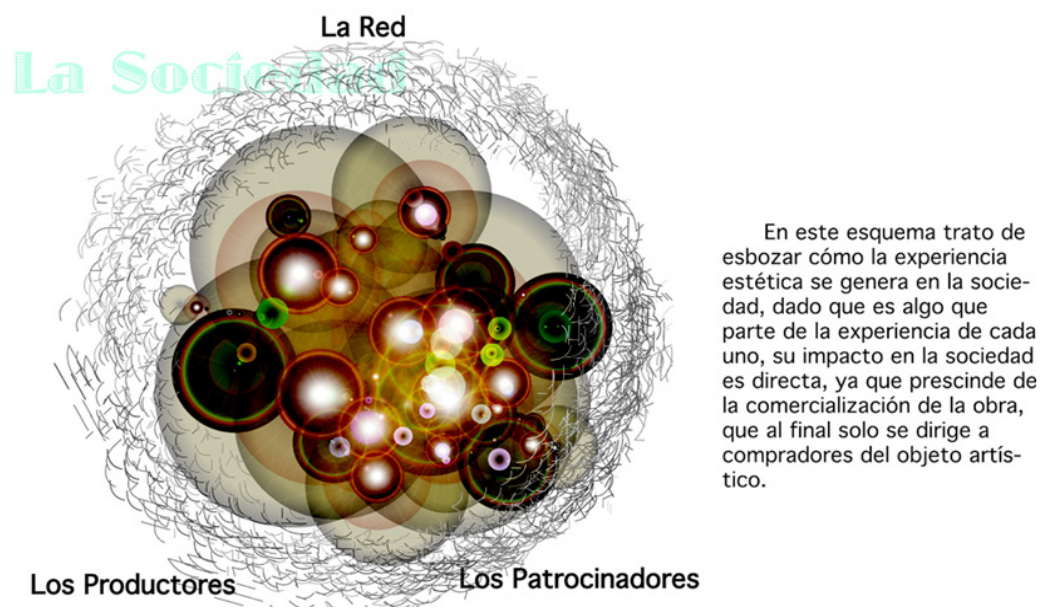

Figura 2. Esquema elaborado personalmente. 
Para aclarar la figura de la experiencia estética, basta con que veamos el proceso de construcción del CSA la Tabacalera y el Campo de la Cebada, que son dos ejemplos que quiero citar en esta tesis. La obra entendida en estos dos casos es un proceso permanente constructivo, formando parte de la experiencia cotidiana de los vecinos. Esta misma, es la liberación de las formas determinadas, y es una experiencia cotidiana donde se muestran las posibilidades de recuperar la autoría de cada sujeto, en la que han habido momentos en los que la construcción en conjunto entre diferentes sujetos ha sido posible.

\subsection{El CSA la Tabacalera}

La Tabacalera es un centro social autogestionado. En la Tabacalera se sostienen criterios metodológicos básicos y la cultura procomún: Autogestión, apertura a toda clase de agentes sociales, cooperación, generación de recursos comunes de uso público, transparencia y accesibilidad, corresponsabilidad, cultura libre, evaluación continua -interna y externa- del proceso, prioridad de los procedimientos sobre las formas o resultados culturales concretos y prevalencia de parámetros de protección de los pequeños frente a los grandes, la autoproducción frente a la producción mercantil, etc.

LTBC apuesta por una forma de producción cultural donde el valor añadido es el modo de producción que no escinde las fases del proceso: financiación-contexto-espacioproducción-obra-valor-exhibición-reproducción forman un todo. La producción aúna el arte y su contexto, los intersecciona y mezcla, para que se retroalimenten. El valor del trabajo, de la obra, proviene de un trazado que se compone de múltiples relaciones y capas de relación. Cada paso o cada fase, que no están predeterminados ni "asegurados" por un servicio, obligan a pensar, organizarse y cooperar. Lo generado así resulta, entonces, radicalmente distinto por el cómo y no por el dónde o ni siquiera el qué (el Dossier de CSA la Tabacalera, 2011, p.30).

A continuación se reproducen las citas de algunas entrevistas que hice durante el mes de mayo de 2012 para explicar mejor el concepto sobre la creación colectiva y la artesanía.

Entrevista a Carlos Vidania, usuario del CSA la Tabacalera:

¿Cuál era el objetivo en la ejecución del proyecto?

Carlos Vidania:

Uno de los objetivos de la Tabacalera es aprender cómo se gesta un proceso democrático sin experiencia previa, sin conocerse, sin tener un acuerdo común inicial. Todo ese proceso complejo y lleno de tensiones forman parte del proyecto. Es decir, cómo establecer un mecanismo democrático de decisión cuando hay resistencias de democracias, cómo hacerse transparente cuando no hay cultura de transparencia, cómo hacer arte colaborativo cuando todo el mundo piensa que lo importante del arte es la firma de lo mismo, o cómo hacer la cultura libre cuando todo el mundo piensa en la cultura del mercado (Carlos Vidania, comunicación personal, 30 de abril, 2012). 
¿Dirías que se ha generado la sostenibilidad social en este proceso de construcción? Carlos Vidania:

La Tabacalera históricamente fue un edificio muy importante en este barrio, es la fábrica más grande de Lavapiés, y no solo producía cigarritos, producía una forma de relación social, eran otros tiempos del trabajo, y además, la mayor parte, hasta el 90\% de plantilla eran mujeres, que sostenían económicamente el barrio, la familia y otras formas de relaciones humanas. (...). Por un lado, es establecer un hilo entre aquello que fue tan significativo dentro del barrio, generando un proyecto que cumpla hasta cierto punto la misma función, de constructores de relaciones, incluso la dinamización de la vida social. Por otro lado, es mantener la intervención de capital humano y no económico en el edificio, considerando que la mesura económica guía la comprensión del funcionamiento económico y la sostenibilidad humana.

El grupo de autoconstrucción, que era uno de los ejes de trabajo vinculaba esta propuesta que ya habíamos hecho a procesos de reparación y al tratamiento del edificio, y que demostraran que se puede tener un edificio abierto, público con el mínimo coste, y con un valor mayor que si se reformase entero (Carlos Vidania, comunicación personal, 30 de abril, 2012).

Entrevista a Jordí Claramonte, usuario del CSA la Tabacalera:

¿Cuál es la utilidad social de este centro social?

Jordí Claramonte:

Ahora estamos en un momento de cambio en el que tenemos que plantearnos cuál es la función de este centro social en esta ciudad y en este barrio. Para ello, vamos a empezar y hemos empezado proyectos productivos que dan trabajos a la gente, que de alguna manera se meten ya en las formas de vivir de la gente. Lo que hemos decidido en la asamblea de economía, es que no vamos a centrarnos de nuevo en el valor del dinero, ni en producir dinero para pagar el sueldo de la gente, sino que vamos a intentar pensar en dos direcciones: la dirección de la comunidad y la dirección de las necesidades. ¿Qué necesitamos?, ¿cómo podemos conseguir lo que necesitamos? (....) La Tabacalera, porque hay dos plantas superiores que están vacías, puede proporcionar viviendas, puede proporcionar la capacidad de organizar la comida en grandes cantidades (proveniente del campo o de la basura), y dar de comer a la gente, puede proporcionar el acceso a la cultura, puede proporcionar el transporte (tenemos un taller de bici fantástico), y puede de alguna manera atender a esas necesidades, bajo la perspectiva de una comunidad. Es decir, de grupos autoorganizados, que regulen sus propias necesidades. (Jordí Claramonte, comunicación personal, 7 de mayo, 2012).

Respecto a la pregunta de si existe un estado de equilibrio entre la creación, la producción, la valoración y la financiación, Christian Salazar y Carmen Gloria, dos usuarios de Nave Trapecio, la nave de la producción de herramientas, me ofrecieron otra manera de ver esta relación que tiene que ver con el carácter de gratuidad de la Tabacalera:

Cristian Salazar: 
Digamos que el aporte más grande de este centro es que es un contenedor de la cultura libre y genera cultura libre. Es un espacio donde se pueden materializar X cosas, se puede obtener un conocimiento, construir una red de trabajo, o llegar a conocer a la persona con la que vas a empezar un vínculo de trabajo (Cristian Salazar, comunicación personal, 24 de abril, 2012).

Carmen relaciona las raíces de la palabra "gratuidad" con la "gratitud" (agradecimiento). Podemos entender que cuando el dinero no interviene en la ejecución de estas actividades, se busca otro estado de equilibrio, su fuerza se compensa de alguna manera, pero no tiene por qué restringirse a las actividades del mercado:

Carmen Gloria:

A veces, investigas algo, lo llevas fuera, y esto desemboca en un trabajo remunerado. Después si quieres compensar al centro porque usas su espacio, lo puedes devolver con tiempo de construcción o reparación. Es buscar otra forma de economía que tiene que ver con el origen de palabra "gratitud" que es 'agradecer", buscando un equilibrio, que no tiene por qué pasar por una economía de mercado. Todo se compensa de alguna manera (Carmen Gloria, comunicación personal, 24 de abril, 2012).

\subsection{El Campo de la Cebada}

El proyecto el Campo de la Cebada comparte la filosofía procomún, afín a la Tabacalera. Se sitúa en La Latina (Madrid) y es un proyecto del centro deportivo autogestionado que surgió después de que fracasara la intervención del Plan de Rehabilitación del Centro Urbano del Ayuntamiento en 2006 por falta de financiación para continuar. En vez de dejarlo abandonado como cualquier descampado en el centro del Madrid, los vecinos han dedicado tiempo para generar vida allí.

Cito algunas entrevistas que hice durante el mes de julio del presente 2012. Creo que el trabajo colectivo que se realizó dio una vuelta a nuestra percepción del tiempo en un escenario.

Manuel Pascual García (colectivo Zuloark):

Cuando empezamos a trabajar en el Campo de la Cebada, en una de las reuniones, el Ayuntamiento nos ofrece cerrar el solar durante tres meses, en los que un equipo municipal se encargue de hacer una obra, y que esté terminado cuando los vecinos entren. Nosotros lo que le ofrecemos es trabajar con unos tiempos distintos, y que el tiempo de la fabricación del objeto "el Campo de la Cebada", sea el tiempo que se puede habitar el campo de la Cebada. No que haya una valla, que impida que la gente participe en la creación del espacio, sino que todo el tiempo en el que se utilice este objeto esté contemplado como la creación de la obra. En este sentido, los tiempos cambian radicalmente, ya no existe una inauguración, sino que todos los días se inaugura algo. Ahora se está inaugurando unos muebles hechos en un taller con la Universidad de Colombia, por la tarde se inaugura un festival de música, mañana se inaugurará otra cosa, todos los días se inaugura algo. Pero no se espera a una gran inauguración. Algo que diga, "Vale, ya está terminado el campo, ahora podéis empezar a habitarlo”, sino que todo el proceso de vivir en la calle es el mismo proceso de construirlo, y no se para en ningún momento porque haya que 
hacer una obra que impida que participen los vecinos. A la vez, este contexto de habitar la obra, de habitar este objeto crítico que está siempre en evolución, permite que los vecinos participen mucho más (Manuel Pascual García, comunicación personal, 13 de julio, 2012).

El CSA la Tabacalera y el Campo de la Cebada son dos ejemplos cercanos. Los modos de crear arte en estos dos espacios procomunes suponen un cambio de modelo para el mundo del arte. Cabe destacar que en ambos casos, si no hubiese sido por los servicios gratuitos de los vecinos y los artistas, no se habría construido esta experiencia para todos. Para autoabastecer, se busca otro sistema de economía, como puede ser el banco de tiempo, trueques e intercambios de servicios.

Esta forma de hacer arte, previamente investigada por Kester (2004), Claramonte (2010) y otros autores, llamada Estética de Diálogo y la Estética Modal, es diferente al estado de la estética en el arte premoderno, dado que sus formas se adaptan a las condiciones actuales. Es decir, el arte creado no sigue una belleza universal y predefinida. La entrada de la percepción estética no es unidireccional para comunicar lo que ha sido experimentado, sino que es una comunicación intersubjetiva en la percepción y comprensión. Solo de esta manera, el público sacia su necesidad de encontrar lo bello en su experiencia.

\subsection{Recorrido histórico de cómo es la muerte y la resurrección del autor}

Es la conciencia de la pérdida de toda esencialidad en esta certeza de sí y de la pérdida precisamente de este saber de sí-de la sustancia como del sí mismo, es el dolor que se expresa en las duras palabras de que Dios ha muerto (Hegel, 1966, p.327).

Hegel anunció la muerte de la autonomía del creador y usó a Díos como una metáfora, se trata de la desaparición de la individualidad en los creadores. Esto indirectamente fue una consecuencia de la introducción del modelo capital en el arte, como por ejemplo, los Salones del arte, que al final produjo la confrontación de lo que era lo ideal y lo real del proceso creativo que tiene cualquier sujeto, perdiéndose así, la autonomía de creador.

La tesis de la creatividad universal de todos los individuos se trasladó al Dada, al arte conceptual, "si yo fuese artista, el arte sería todo lo que anunciara que es", "si mi obra fuera expuesta en instituciones artísticas, esta misma sería una obra artística", posteriormente, este modo de hacer fue absorbido como un mecanismo en la institución del arte.

Joseph Beuys, el pionero de esta práctica política, anunció: "todo el mundo es artista". El arte fue para él un libre desarrollo de la personalidad. Dotando al arte de una utilidad social, Beuys consideró que el arte era un elemento que ayudaba al desarrollo de la creatividad de cada persona. A través de "crear", uno podía realizarse y eso permitía mejorar la sociedad y la educación. Aunque solemos interpretar esto como prácticas políticas, Beuys aclaró que era prácticas artísticas: 
Para mi es una acción artística. Porque ese concepto artístico está pensado para hacer que la autodeterminación que exige la democracia se vea ya como posibilidad. Hay mucha gente que dice "sí, bien, pero el ser humano no puede determinarse a sí mismo, no tiene libertad interna". Así que lo que queremos discutir es una ciencia de la libertad. Queremos partir cada vez más de la autodeterminación, de la libertad humana como punto de partida creativo, o sea artístico. Así que es una cuestión cultural antes que nada (Bodenmann-Ritter, 2005, p.56).

A parte de las prácticas y teorías de Joseph Beuys, la guía de selección en National Endowment for the Arts (la institución más grande en los Estados Unidos que asigna la financiación para que los artistas del arte público puedan realizar sus obras) también favoreció que los artistas se convirtieran en facilitadores de la creación colectiva, dirigiendo el arte al interés público. En 1979, NEA investigó métodos para que el público interactuara con la obra instalada. Ese fue el contexto destacado por Suzanne Lucy sobre New Genre Public Art. La línea de actuación del arte público prosperó aún más en los años 80. En 1983 la NEA convocó a que los destinatarios de la financiación (premio) tuvieran que presentar "los planes de participación, de preparación y el dialogo con las comunidades". En los años noventa, se amplió esta línea de actuación para fomentar la participación comunitaria en actividades educativas y artísticas. (Finkelpearl, 2000, p.43)

Todo esto, nos da una visión de cómo los artistas se convierten en activadores de la creatividad del público. Esta tendencia no solo pasó en el ámbito artístico, sino también en la sociología y la educación, por ejemplo, los métodos que dan poderes constituyentes al público, a Investigación Acción Participativa y a Teatros de los Oprimidos (Boal, 1985).

\section{Análisis sobre la lógica de ejemplos existentes}

En el campo de la práctica, encontramos frutos de creación colectiva que sirven como paradigmas para este tema. Desde el sujeto, examinamos las teorías de la sensibilidad que nos hacen reflexionar sobre las experiencias actuales.

\section{1 La relación entre la percepción del sujeto con los casos}

¿Cómo son los patrones de los espacios procomunes y de las construcciones artesanales? Según Sennett, "un linde ecológico, como una membrana celular, resiste la mezcla indiscriminada; contiene diferencia, pero es poroso” (2009, p.278). Los patrones de espacios procomunes aciertan bastante con los espacios de resistencia como un tipo de "borde", "linde" o "limite entre dos espacios". Consisten en un tipo de tejido caótico y abundante, desarrollado por la conjunción de los habitantes. Este concepto surge creando variadas utilidades entre espacios públicos y recuperando espacios muertos.

Esta ambigüedad pasa en los alrededores de las murallas y las fronteras de las ciudades medievales, que se van transformando en lindes más activas debido a las actividades económicas que se desarrollan alrededor de ellas, por ejemplo, las comunidades multiculturales o los caos de los guetos. 
Rudofsky en su libro "La arquitectura sin arquitecto" confirma que a diferencia de la historia ortodoxa de la arquitectura, que considera exclusivamente la labor del arquitecto, en muchas de las arquitecturas comunales se toman todavía las formas tradicionales y se conserva el acto de construir como una actividad espontánea, entendiendo esto como lo "humano" de la arquitectura. Pietro Beluschi definió la arquitectura comunal como "un arte comunal producido no por unos pocos intelectuales o especialistas, sino por la actividad espontánea y continua de todo un pueblo con una herencia común, actuando en una comunidad de experiencia" (Rudofsky, 1974, prefacio).

Según El lenguaje de patrón de Alexander Christopher (1969), en la cultura inconsciente, los artesanos individuales tienen poco control en el proceso de producción. La "tradición" tiene suficiente poder para que la pauta de adaptación se ajuste a un conjunto y haga que la cultura en su totalidad no se modifique sin rumbo.

Esbozó muchas imágenes sobre análisis formales y campos de fuerza para explicar cómo el patrón de espacio se multiplicaba como si fuese un lenguaje. Pero el resultado no ha sido fructuoso en la sociedad moderna debido a que las construcciones modernas son representaciones de la ideología de arquitectos o de directores de grandes inversiones. En cambio, en la cultura inconsciente (por la falta de grupos directivos), sin darse cuenta de cuestiones de contexto, el fruto de artesanía justifica la obra en su contexto y la integración de la obra con la naturaleza, como por ejemplo, arquitecturas vernáculas.

\section{2 La relación entre la percepción del sujeto con la teoría}

Para llevar a cabo la resurrección de la autoría del público en los espacios procomunes, es necesario recurrir a los puntos que previamente han sido discutidos por autores de renombre, como Schiller, Hegel y Dewey, en el ámbito de la experiencia estética. Tal como ellos describían, la acción de "crear" daba razón al libre desarrollo de la personalidad, uno podía realizarse y conseguir la libertad para auto determinarse.

En la conclusión del libro Extranjero (en el Artesano), Sennett citó el libro de John Dewey para exponer su visión, "el espiritu artesanal tiene un hogar filosófico en el pragmatismo" (2009, p.351). En la labor de la artesanía, no se separan las prácticas y las emociones.

Esta práctica de artesanía da bastante sentido al "qué hacer", la unión del pensamiento y la práctica, fue entendida por los teóricos premodernos como el genio. Dewey anunció lo siguiente en El Arte como Experiencia: cuando la participación viene después de una fase de desconexión y conflicto, lleva dentro de sí mismo los gérmenes de una consumación próxima a lo estético (2008, p. 16). Según el autor, el arte se basa en la agencialidad de las relaciones que se crean en un proceso, y es autónomo del mercado de arte y de las instituciones. Todo el mundo puede lograr una experiencia estética, dominada por los modos de relación que se genera en la actuación de un sujeto con su entorno.

De este modo, la experiencia estética nos permite entender el arte como producción y percepción. Se acerca a la cotidianeidad y se integra en las experiencias. Este arte es intransferible, goza de su autonomía en el tiempo y está libre de formas. 
Cuando algún material encuentra un medio que expresa su valor en la experiencia - es decir, su valor imaginativo y emocional-, se hace sustancia de una obra de arte. La lucha permanente del arte consiste en convertir materiales tartamudos o mudos en la experiencia ordinaria, en medios elocuentes. Recordando que el arte denota una cualidad de la acción y de las cosas hechas, toda auténtica obra nueva de arte es, en cierto grado, por sí misma, el nacimiento de un arte nuevo (Dewey, 1934/2008, p. 257).

Esa autonomía del arte que Dewey revela, determinada por la experiencia propia del autor y el tiempo, es una reflexión curiosa que se refleja bastante en el comentario que hizo Pedro Herrero, artista y usuario del Campo de la Cebada:

Pedro Herrero:

Creo que el hecho artístico está en circunstancias del espacio, de economía, de otras cosas. Luego, la persona que entre por el portón, se enganche, porque este espacio tiene una estructura extraña, pero autoconstruida, y esto todo le llama a la atención. Es ese "todo" en conjunto, el hecho artístico, ecológico, cultural, arquitectónico, llaman a ciudadano que entre aquí, y creo que se lleva otra manera de ver la ciudadanía u otra manera de ver la gestión de las cosas. (...). Aquí, sin darte cuenta, participas en un montón de cosas, el que está regando ahora mismo está participando en el teatro sin querer, lo mismo que el que está viendo la obra, luego se pasa por aqui y ve lo que está ocurriendo aqui en el huerto (Pedro Herrero, comunicación personal, 7 de julio, 2012).

Schiller (1759 - 1805) lanzó el término "la educación estética" después de dos hechos históricos, la revolución francesa y la revolución inglesa a finales del siglo XVIII. Sentía necesario proponer eso, considerando la estética como solución de la ruptura de la humanidad.

Ante todo, cabe mencionar que el estado estético que Schiller considera, incluye el estado, el ser de un sujeto y sus percepciones sobre el mundo. El estado cambia y está limitado por el tiempo, en cambio, el ser tiene la libertad de mantenerse en permanencia o cambiar por su propia voluntad. En base a eso, Schiller usa el término, "el impulso del juego" para determinar momentos en los que el impuso sensible reacciona en base a toda presencia sensible inmediata. Esto se une al impulso formal en un estado. "el impulso de juego se encaminaría a suprimir el tiempo en el 'tiempo', a conciliar el devenir con el ser absoluto, la variación con la identidad" (Schiller, 1990, p. 225). Esto se podría comparar al momento en el que sujeto llega al auge de su satisfacción, a través de su percepción y creación por medio de una experiencia.

Hegel anunció la muerte de Díos como una metáfora de la muerte del creador por la pérdida de la cualidad Genio, que representaba una concordancia entre el deseo personal con la etnicidad. Pero esto se perdió en la sociedad moderna debido a que surgió la individualidad libre.

En la vida de un pueblo (premoderno), la costumbre, la sustancia ética, forman parte de la cultura, eran razones conscientes entre sí, tanto auto consciencias reales como la consciencia universal y de su propio ser. 
En un pueblo libre se realiza, por tanto, en verdad la razón; ésta es el espíritu vivo presente, en que el individuo no sólo encuentra expresado su destino, es decir, su esencia universal y singular, y la encuentra presente como coseidad, sino que él mismo es esta esencia y ha alcanzado también su destino. De ahí que los hombres más sabios de la antigüedad hayan formulado la máxima de que la sabiduría y la virtud consisten en vivir de acuerdo con las costumbres de su pueblo (Hegel, 1966, p. 157-158).

Esta afirmación da pie a entender que nunca más se podrá volver a ese estado de cualidad genuina en el hombre moderno, pero en el apartado "el Reino Animal del Espíritu y el Engaño, o la Cosa Misma" Hegel confirma que la individualidad se reconoce a sí misma por sus acciones. Esto hace referencia al estado de "obrar", que es cuando la autoconsciencia se convierte por sí misma en la realidad, dirigida así por su impulso natural: "placeres y necesidades".

Por tanto, el individuo sólo puede experimentar el goce de sí mismo, puesto que sabe que en su realidad no puede encontrar otra cosa que su unidad con él, es decir, solamente la certeza de sí mismo en su verdad y que, por tanto, siempre alcanza su fin (Hegel, 1966, p. 178).

\section{Conclusiones}

Los espacios procomunes recuperan el fundamento de los trabajos colectivos en esta sociedad debido a su carácter de gratuidad, su función como taller y como contenedor de cultura libre.

Hablamos de la resurrección del público en la autoría en los espacios procomunes, porque en estos proyectos de autoconstrucción, un individuo se retroalimenta de su propia contribución, basándose en la experiencia recíproca de crear y percibir. Esta experiencia estética se genera a partir de la vivencia de cada autor, por lo tanto, es un arte único para cada cual y lleva implícito su autonomía. Basándose en la ideología de que todo el mundo puede ser artista, este modo de adquirir la experiencia estética recupera el espíritu del artesano: homo faber, que reflexiona sobre el libre desarrollo de la personalidad y de acción de obrar, de crear la unión de práctica y pensamiento y experimentar el goce de sí mismo.

Los casos y las teorías presentadas en este artículo componen un paradigma de este tema. En base a esto, más adelante, podremos discutir sobre la praxis, la aclaración de la identificación de los casos investigados, los foros de indicadores relativos y la metodología instituyente y creativa a través de prácticas artísticas, como la comprobación de este paradigma, con el fin de crear una epistemología para la gente local.

\section{Referencias}

Alexander, C.W. (1969). Ensayo sobre la síntesis de la forma. Buenos Aires: Infinito. Bodenmann-Ritter, C.(2005).Joseph Beuys: Cada hombre, un artista: Conversaciones en Documenta 5 - 1972. J. L. Arántegui \& B. Monte (Trad.). Madrid: A. Machado Libros. 
Chin, M. (14 de diciembre, 2010). Transdisciplinary lecture: Mel Chin, artista. [seminario de vídeo]. The New School. Nueva York. Recuperado de: http://www. youtube.com/watch? $v=$ VnibKAEJpoE\&feature=endscreen

Collins, T. M. (2007). Art ecology and planning: Strategic concepts and creativity within the Post industrial public realm. Tesis doctoral. la Universidad de Plymouth.

Dewey, J. (2008). El arte como experiencia. J. Claramonte (Trad.). Barcelona: Paidós, D. L. (primera publicación en 1934).

Dossier de CSA la Tabacalera. (2011). Recuperado en http://latabacalera.net/wpcontent/uploads/2011/11/Dossier-CSA-La-Tabacalera.pdf

Finkelpearl, T. (2000). Diologues in public art : Interviews with Vito Acconci, John Ahearn ... et al. Cambridge: The MIT Press.

Garrido, M. (2007). Prólogo. La tercera aventura crítica de Kant. En I. Kant, Crítica del juicio. Madrid : Tecnos, D.L.

Hegel, G. W. F. (1966). Fenomenología del espiritu. W. Roces (Trad.). México, D.F., Fondo de Cultura Económica.

Martínez, C. (2012). How a tedpole becomes a frog, belated aesthetics, politics, and animated matter: Toward a theory of artistic research. En C. C. Bakargiev, C. Martinez \& F. Berardi (Coord.), Documenta 13: Catalog I/3, The book of books (pp. 46-57). Ostfildern: Hatje Cantz Verlag.

Rahman, M. A. \& Borda O. F. (2009). La situación actual y las perspectivas de la investigación-acción participativa en el mundo. En M. C. Salazar (Coord.), La investigación-acción participativa, inicios y desarrollos, Kurt Lewin, Sol Tax, Rodolfo Stavenhagen, Orlando Fals Borda, León Zamosc, Stephen Kemmis, Anisur Rahman. Madrid: Editorial Popular (primera edición en 1992).

Rudofsky, B. (1973). Arquitectura sin arquitectos : Breve introducción a la arquitectura sin genealogía, Buenos Aires: Eudeba.

Sennett, R. (2009). El Artesano. M. A. Galmarini (Trad.). Barcelona: Anagrama.

Schiller, F. (1990). Kallias : Cartas sobre la educación estética del hombre. J. Feijóo \& J. Seca (Trad.). Barcelona: Anthropos; Madrid: Centro de Publicaciones del MEC.

Villasante, T. R. (2002). Sujetos en movimiento, redes y procesos creativos en la complejidad social - construyendo ciudadanía/4. Montevideo: NordanComunidad Del Sur. 
